pipette. It is always as well to have a few spare "washers" in the cystoscope box, for when the sheath is being sterilised this washer in the screw cap is apt to boil out and is lost. The cable attachment to the cystoscope should point upwards so as not to be in the way of the "sheath" during manipulation. Gum elastic tubing has been tried in place of the rubber but the latter is more suitable. A spring arrangement for grasping the catbeter while it is being pushed onwards in order that the fingers do not come in contact with it at all was too complicated. In lieu of this the hands must, of course, be efficiently sterilised before touching the catheter. The illustration also shows Mr. John Pardoe's most excellent eye-piece fitted on to the cystoscope.

The "sheath" may be obtained from Mr. J. H. Montague, 69, New Bond-street, W.

$$
\text { R. J. WILlan, M.B., B.S. Durh., }
$$

Senior House Surgeon, St. Peter's Hospital for Stone, London.

\section{A NEW INSTRUMENT FOR TREATING GLEET.}

For some six years I have successfully used a method of treating chronic urethritis, chiefly where the follicles are engaged, and $I$ can recommend it to others where ordinary methods fail. This method embraces (1) the emptying of the follicles by aspiration and the passage of large bulbs; and (2) when they are empty and kept patent the injection of certain solutions into them by means of a large injector. Follicular urethritis is common, especially in the prostate, and its treatment is difficult and uncertain. Tersely, the enigma presented is to get the secretion out of, and the injection into, the follicles and utricle. Up to the present time massage, the passage of large sounds, Kollman's dilator, and sometimes the obliteration of the follicles of the penile urethra by caustic or electric action were the aids possessed by surgeons. The first three are often inefficient, while the last two are of only occasional utility and are always difficult. At first I used Erichsen's syringe, to which I attached a small
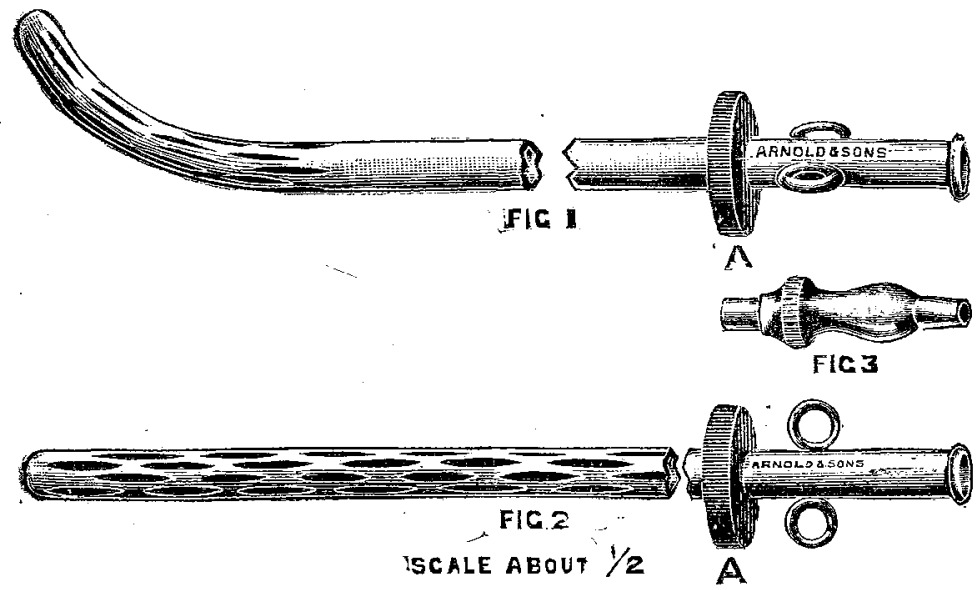

aspirator. It was too small and the metal between the holes pressed transversely on the ducts. The instruments represented in the accompanying figures are much more effectual; Fig. 1 is for the deep urethra and Fig. 2 is for the anterior urethra; Fig. 3 is a connexion for an aspirator. The perforations are oblong. $A$ and $A$ are indiarubber discs which travel on the instruments and block the meatus. Barred instruments may be used. Having well smeared them with liquid paraffin the particular one selected is introduced, an aspirator or exhausting syringe is applied, and an intra-urethral negative pressure is slowly and gently created. The urethra is then flushed and swept clear of its secretion by a large bulb shaped like a turkey's egg. The injection is then used through these instruments or other large injectors. I wait some time after injecting so as to allow the spasm which grasps the instrument to subside. I am much indebted to Messrs. Arnold and Sons, West Smithfield, London, F.C., for carrying out my suggestions. The instruments are made of silver.

Whilst on the subject of aspiration I may offer to others an aid which has frequently been of good service to me. I refer to drainage of the bladder by means of aspiration, whether this be suprapubic or otherwise. I make use of a resistant indiarubber tube with a tap to connect the catheter to a large bottle, the air of which is occasionally exhausted by a Potain apparatus. The tap is now and then turned so as to aspirate the urine away, thus keeping the bladder empty. I have found this device more effectual than even Cathcart's ingenious plan of syphonage.

Finsbury-pavement, E.C.

James MacMun.

\section{THE FIRST FRENCH CONGRESS OF STOMATOLOGY.}

TuE Société de Stomatologie, wishing to celebrate the twentieth year of its foundation, has decided to organise a Congress of Stomatology in Paris which will be held from Angust 1st to 5th, 1907. It is stated that this Congress is intended to demonstrate that stomatology is a branch of medicine-a medical and surgical specialty-just as much as is ophthalmology or laryngology, and that it requires from those who practise it a complete knowledge of medicine. The diseases of the mouth, teeth, and jaws, it is urged, are closely related to other diseases and to general pathology, and a correct knowledge of this relation is necessary for all who practise stomatology with all that it includes.

In addition to many papers and demonstrations promised by the leading members of the Société de Stomatologie a number of foreign medical men have promised to take an active part in the proceedings. Several distinguished dentists from America, including Professor Talbot (Chicago) Dr. Latham (Chicago), Dr. Brown (Milwaukee), Dr. Fletcher (Cincinnati), and Dr. Rhein (New York), have already promised to give papers or demonstrations. A museum of all the various models, instruments, pathological specimens, \&c., which are of use and interest to the dental surgeon is being formed, and it is hoped that England will also contribute largely to the success of this department. The names of M. Cruet, president, and M. Galippe and M. Redier, honorary presidents, appear on the committee of organisation. M. Chompret, 182, Rue de Rivoli, Paris, is the secretary, from whom all information can be obtained.

Immediately following this congress (August 6th and 7th the International Stomatological Association, having the same objects, will hold its first meetings and will occupy itself with questions relating to legislation and professional organisation, education, oral hygiene, and international stomatological bibliography. The committee of organisation of this association is formed of one representative from each country and includes several well-known names, such as those of Professor J. Arkövy of Budapest, M. Cruet of Paris, Dr. H. Allaeys of Antwerp, and Professor Eugene Talbot of Chicago. Dr. J. Sim Wallace of London represents England.

Membership of the Congress of Stomatology is open (1) to all members of the Société de Stomatologie de Paris, whether charter or regular members ; (2) to all legally qualified medical men practising oral or dental surgery-including, therefore, medical graduates of a French or foreign uni. versity or the possessors of the diplomas of the Royal Colleges of Great Britain and Ireland; and (3) to individuals who by reason of their work, knowledge, or attainments. may be admitted by the committee of organisation. Meetings will be held in the amphitheatre of the Faculty of Medicine and the proceedings will include four discussions upon stomatological questions; communications on any subject in stomatological science, mechanical dentistry included, may also be presented. Patients, specimens, drawings, casts, and instruments will be shown. The membership subscription is 25 francs. French will be the official language of the congress but Esperanto will be admitted in the discussions; Finglish and German will also be admitted if followed by a French summary of the lecture or discussion.

Medical Defence Union.-The annual general meeting of the Medical Defence Union will be held at the Midland Hotel, Manchester, on Thursday, May 16th, at 4 P.X., to receive the annual report of the council and the statement of accounts, together with the auditor's certificate for the year 1906 ; to elect three members of council, Mr. J. Roche Lynch, Dr. A. A. Mackeith, and Dr. F. J. Wethered, honorary treasurer, retiring by ballot under Rule 28 , but offering themselves for re-election; to appoint an auditor and to fix his remuneration; and to vote for the payment of railway fares as expenses incurred by provincial members of the council attending council meetings. 